\title{
Comparison of the pattern of atrophy of the corpus callosum in frontotemporal dementia, progressive supranuclear palsy, and Alzheimer's disease
}

Hiroshi Yamauchi, Hidenao Fukuyama, Yasuhiro Nagahama, Yukinori Katsumi, Takuya Hayashi, Chisako Oyanagi, Junji Konishi, Hideo Shio

\begin{abstract}
Objectives-The loss of the neurons in layer 3, one of the groups of cortical neurons most vulnerable in various degenerative brain diseases, results in axonal degeneration leading to atrophy of the corpus callosum. Previous studies showed callosal atrophy in three degenerative dementias: frontotemporal dementia (FTD), progressive supranuclear palsy (PSP), and Alzheimer's disease (AD). However, it is unclear whether a characteristic pattern of atrophy is present in each. The objective of this study was to investigate whether the pattern of the callosal atrophy was different among patients with FTD, PSP, or early onset AD.
\end{abstract}

Methods-Eleven patients with FTD, nine patients with PSP, 16 patients with early onset $\mathrm{AD}$, and 23 normal controls, all age and sex matched, were studied using MRI. The ratios of midsagittal corpus callosum areas to the midline internal skull surface area on $\mathrm{T} 1$ weighted images were analyzed. The corpus callosum was divided into quarters: the anterior, middleanterior, middle-posterior, and posterior portions.

Results-Compared with controls, all three patient groups had significantly decreased total callosal/skull area ratio. An analysis of covariance adjusted for the total callosal area/skull area ratio showed that the anterior quarter callosal/skull area ratio in FTD, the middle-anterior quarter area ratio in PSP, and the posterior quarter area ratio in $\mathrm{AD}$ were significantly smaller than those in the other three groups.

Conclusion-Although atrophy of the corpus callosum is not specific to any degenerative dementia, the patterns of the atrophy are different among patients with FTD, PSP, or early onset AD. Differential patterns of callosal atrophy might reflect characteristic patterns of neocortical involvement in each degenerative dementia. (F Neurol Neurosurg Psychiatry 2000;69:623-629)

Keywords: frontotemporal dementia; progressive supranuclear palsy; Alzheimer's disease; corpus callosum
In the evaluation of dementia, structural imaging by CT or MRI may be useful for detecting differential patterns of cortical involvement in each disease, because regional cortical pathology, especially neuronal loss, may be reflected by regional cortical atrophy. In clinical practice, the qualitative evaluation of the pattern of regional cortical atrophy can provide additional clues for the differential diagnosis of dementia. However, mild neuronal loss in the cerebral cortex may not necessarily be reflected by cortical atrophy due to reactive gliosis. Furthermore, the quantitative verification of differential patterns of cortical atrophy throughout the entire cerebral cortex is not feasible. ${ }^{1}$

Atrophy of the corpus callosum, the quantitative evaluation of which is easy on midsagittal MR images, may be an indirect but sensitive index of cortical neuronal loss associated with cognitive impairment in degenerative dementias. Pathological studies of degenerative dementias have disclosed neuronal loss or appearance of neurofibrillary tangles in layer 3 of the cerebral cortex, ${ }^{2}$ suggesting that the third layer is one of the groups of cortical neurons most affected in these diseases. Layer 3 includes the pyramidal cells that project long association fibres connecting intrahemispheric cortical regions and commissural fibres connecting bilateral cortical regions through the corpus callosum. ${ }^{3}$ Therefore, damage to layer 3 in degenerative dementias would impair large scale networks among cortical regions that are indispensable to the performance of complex cognitive functions. ${ }^{4}$ The extent of the atrophy of the corpus callosum, which might parallel the overall loss of long association and commissural fibres, may thus reflect the degree of disconnection among cortical regions, potentially contributing to the impression of neocortical dysfunction in degenerative dementias. In addition, the regional distribution of callosal atrophy may reflect a characteristic pattern of the cortical damage, which would cause characteristic impairment of cortical functions in each disease. The topographical location of the interhemispheric fibres in the corpus callosum may be arranged along its rostral-caudal extent in parallel with the site of origin of the fibres along the anterior to posterior hemispheric axis. ${ }^{5}{ }^{6}$ Thus, the pattern of callosal atrophy may be a useful morphological index showing the relative predominance of anterior or posterior cortical involvement correspond- 
ing to the respective pattern of cognitive dysfunction in degenerative dementias.

Frontotemporal dementia (FTD), progressive supranuclear palsy (PSP), and Alzheimer's disease (AD) are neurodegenerative diseases which present with dementia. Frontotemporal dementia is the third most common cause of degenerative cortical dementia of presenile onset and is often mistaken for AD. ${ }^{7-9}$ Frontotemporal dementia and PSP are characterised by different degrees of frontal lobe dysfunction compared with AD. ${ }^{8}{ }^{10}$ Previous studies showed that atrophy of the corpus callosum is present in patients with FTD, PSP, or AD compared with normal control subjects, and that it is associated with cognitive impairment and cerebral cortical hypometabolism. ${ }^{11-13} \mathrm{Al}-$ though atrophy of the corpus callosum may not be specific to any degenerative dementia, the pattern of its atrophy may be characteristic for each disease. However, differences of the pattern of callosal atrophy among degenerative dementias have rarely been explored. Frontotemporal dementia typically affects prefrontal and anterior temporal regions, whereas $\mathrm{AD}$ tends to involve more posterior temporal and parietal areas. ${ }^{14}$ Neurofibrillary degeneration in the cerebral cortex in PSP has recently gained widespread recognition, and pathological changes may be prominent in the posterior frontal region among neocortical regions. ${ }^{15-17}$ Thus, the severity of the anterior predominance of callosal atrophy may show the following order: FTD $>$ PSP $>$ AD. The objective of this study was to investigate whether the pattern of the atrophy of the corpus callosum is different among patients with FTD, PSP, or early onset $\mathrm{AD}$.

\section{Subjects and methods}

SUBJECTS

Patients with FTD, PSP, or AD were evaluated at our university hospital. Table 1 shows their demographic data. Diagnostic evaluations included a detailed history, neurological evaluation, standardised neuropsychological assessments, MRI, and PET or SPECT. Diagnosis was determined on the basis of clinical symptoms and signs, and MRI, and PET or SPECT data were not used as inclusion criteria but as supporting findings. The cognitive function was screened with the mini mental state examination (MMSE). ${ }^{18}$ Eleven right handed patients with FTD met research criteria for the diagnosis of FTD developed by the Lund and Manchester Groups. ${ }^{78}$ The age of onset was 65

Table 1 Patient data

\begin{tabular}{llllll}
\hline & Controls & FTD & PSP & AD & p Value \\
\hline No of patients (M/F) & $23(15 / 8)$ & $11(7 / 4)$ & $9(6 / 3)$ & $16(10 / 6)$ & NS $^{\star}$ \\
Age (y) & $61(6)$ & $61(11)$ & $65(3)$ & $61(3)$ & NS \\
(range) & $(50-69)$ & $(31-68)$ & $(60-69)$ & $(55-64)$ & $<0.001$ \\
MMSE score & $29(1)$ & $22(6)$ & $21(4)$ & $19(5)$ & \\
& $(27-30)$ & $(7-29)$ & $(14-28)$ & $(5-27)$ & $<0.02$ \\
Duration (months) $\dagger$ & & $22(16)$ & $40(20)$ & $22(9)$ & NS \\
& & $(6-60)$ & $(19-72)$ & $(11-48)$ & $11(3)$ \\
Education (y) & $11(3)$ & $12(2)$ & $12(4)$ & $(6-16)$ & \\
& $(7-16)$ & $(8-16)$ & $(8-16)$ & $(6)$ & \\
\hline
\end{tabular}

Values are mean (SD). p Values are for univariate ANOVA except as noted.

${ }^{\star} \chi^{2}$ test for sex distribution across groups.

†Duration of dementia for FTD and AD, of parkinsonism for PSP. or younger in all patients. Five patients had amyotrophic lateral sclerosis (ALS), the diagnosis of which was supported by the EMG findings. Two patients are deceased and had pathological confirmation of FTD with ALS. Bilateral frontal hypometabolism on PET or SPECT was present in all cases of FTD. We studied nine right handed patients with PSP matched for age and sex with the patients with FTD. They satisfied the NINCDS-SPSP diagnostic criteria for "probable PSP". ${ }^{19}$ All had gradually progressive parkinsonism, vertical supranuclear palsy with downward gaze abnormalities, and postural instability with unexplained falls. The response to levodopa and dopamine agonists was poor in all cases. Two patients are deceased and had pathological confirmation of PSP. Atrophy of the midbrain on MRI and frontal hypometabolism on PET were present in all patients with PSP. We also studied 16 right handed, age and sex matched patients with early onset $\mathrm{AD}$; the age of onset was younger than 65. All fulfilled the NINCDS-ADRDA diagnostic criteria for "probable Alzheimer's disease". ${ }^{20}$ None had clinical features suggestive of dementia with Lewy bodies. Medial temporal atrophy on MRI and parietotemporal hypometabolism on PET were present in all patients with $\mathrm{AD}$. In all patients, the possibility of space occupying lesions and cerebrovascular lesions was excluded using MRI. Only a few high intensity spots in the subcortical white matter were depicted on T2 weighted MR images. None of them had any history of medical or psychiatric disorder, including encephalitis, hypertension, or diabetes mellitus. All patients had normal laboratory test results including those for syphilis serology, vitamin B12 concentrations, and thyroid hormone concentrations. Two patients with FTD and six with PSP were participants in previously reported studies. ${ }^{12} 13$

We also studied 23 age and sex matched right handed control subjects using MRI. They included five normal subjects who underwent a neurological examination including MRI for the detection of asymptomatic brain disease and 18 subjects who underwent MRI because of headache or dizziness. All of these subjects showed normal neurological findings and no specific neurological disorders other than tension-type headache. None exhibited any abnormal MRI findings, except for a few high intensity spots in the subcortical white matter depicted on T2 weighted images. They also had no history of medical or psychiatric disorder, including hypertension or diabetes mellitus.

MAGNETIC RESONANCE IMAGING

Brain MRI was performed with a Signa unit (General Electric, Milwaukee, WI, USA) operating at a field strength of 1.5 Tesla. T1 weighted sagittal and axial images were obtained using a spin echo pulse sequence (repetition time $400 \mathrm{~ms}$; echo time $15 \mathrm{~ms}$ ). Axial T2 weighted images were also obtained with spin echo pulse sequences (repetition time $3000 \mathrm{~ms}$; echo time $100 \mathrm{~ms}$ ). The slice thickness was $3 \mathrm{~mm}$ for sagittal images and 5 
Table 2 Mean area/skull area ratios (\%) of the four regions of the corpus callosum in patients and controls

\begin{tabular}{llllll}
\hline & Anterior & $\begin{array}{l}\text { Middle } \\
\text { Anterior }\end{array}$ & $\begin{array}{l}\text { Middle } \\
\text { Posterior }\end{array}$ & Posterior & Total \\
\hline Controls $(\mathrm{n}=23)$ & $1.47(0.15)$ & $0.78(0.11)$ & $0.65(0.11)$ & $1.46(0.18)$ & $4.36(0.44)$ \\
FTD $(\mathrm{n}=11)$ & $0.87(0.15)^{\star} \dagger$ & $0.41(0.10) \ddagger$ & $0.37(0.09)$ & $1.06(0.25)$ & $2.70(0.49)$ \\
PSP $(\mathrm{n}=9)$ & $1.09(0.16)$ & $0.47(0.10)$ & $0.43(0.11)$ & $1.21(0.19)$ & $3.20(0.49)$ \\
AD $(\mathrm{n}=16)$ & $1.24(0.26)$ & $0.57(0.13)$ & $0.45(0.15)$ & $0.95(0.19)$ & $3.20(0.55)$ \\
\hline
\end{tabular}

Values are mean $(\mathrm{SD})$.

$\mathrm{FTD}=$ Frontotemporal dementia; $\mathrm{PSP}=$ progressive supranuclear palsy; $\mathrm{AD}=$ Alzheimer's disease ${ }^{\star} \mathrm{p}<0.05 v \mathrm{PSP} ; \mathrm{t} \mathrm{p}<0.001 v \mathrm{AD} ; \neq \mathrm{p}<0.05 v \mathrm{AD}$.

Only the posterior region of patients with PSP showed no significant difference $(p>0.05)$ compared with the controls $(\mathrm{p}<0.001$ for all the other comparisons, ANOVA and post hoc Sheffe's analysis). anterior, middle-anterior, middle-posterior, and posterior portions. In practice, each MR image was recorded with the inferiormost points of both the splenium and the rostrum on the straight inferior margin of the screen of the image analyzer. Then, the points on the border of the corpus callosum with minimum and maximum values of the abscissa on the screen were manually selected as the anterior border of the rostrum and the caudal end of the splenium, respectively. By dividing the difference between the values of the abscissa of these two points into quarters, three additional perpendicular lines were automatically located, dividing the corpus callosum into four parts. The total and regional areas of the corpus callosum were measured, and the absolute values were calculated using a reference scale for MR images. Because persons with a small skull may show a small corpus callosum, ${ }^{21}$ to control for variation in skull size we measured the areas of the midline internal skull surface by manually tracing the line through the inner table, foramen magnum, clivus, sellar diaphragm, and jugum sphenoidale, and we then calculated the total and regional callosal area/skull area ratios. ${ }^{21}$ Although to this end a callosal volume/ skull volume ratio is preferable, we chose the area ratio as the measurements are more easily obtained. All measurements were performed by one investigator who was blinded to the clinical status of the patients.

Before this study, the reliability of our procedure was evaluated by comparing the data of measurements in 20 subjects with and without neurological diseases. For the assessment of intrarater reliability, one rater measured the areas twice. This rater performed the measurements for this study. Interrater reliability was assessed by comparing the measurements by this rater with those by a second rater. For all the measurements of total and partial callosal area/skull area ratios, there were high intrarater and interrater reliabilities (a mean \% difference; less than 1.8 and $2.1 \%$, respectively). The scan-rescan reproducibility was not performed.

\section{STATISTICAL ANALYSIS}

Repeated measures analysis of variance (ANOVA) was performed for the partial area measurements of the corpus callosum to assess whether there was a difference between patients and controls across regions. Then, to compare the callosal area/skull area ratios of the patients and controls, we used one way ANOVA and post hoc Sheffe's analysis to detect regional differences. To determine whether the pattern of callosal atrophy was different among the three patient groups and controls, we used an analysis of covariance (ANCOVA) adjusted for the total callosal area/skull area ratio with contrast methods to detect regional differences. The level of significance was regarded as $p<0.05$. A Bonferroni correction for multiple regional measures was not performed, as this was a pilot study. Multiple linear regression analysis was used to test the independent predictive value of callosal atrophy for cognitive performance. We applied this analysis to the scores of the MMSE as the dependent variable and the patients' age, 


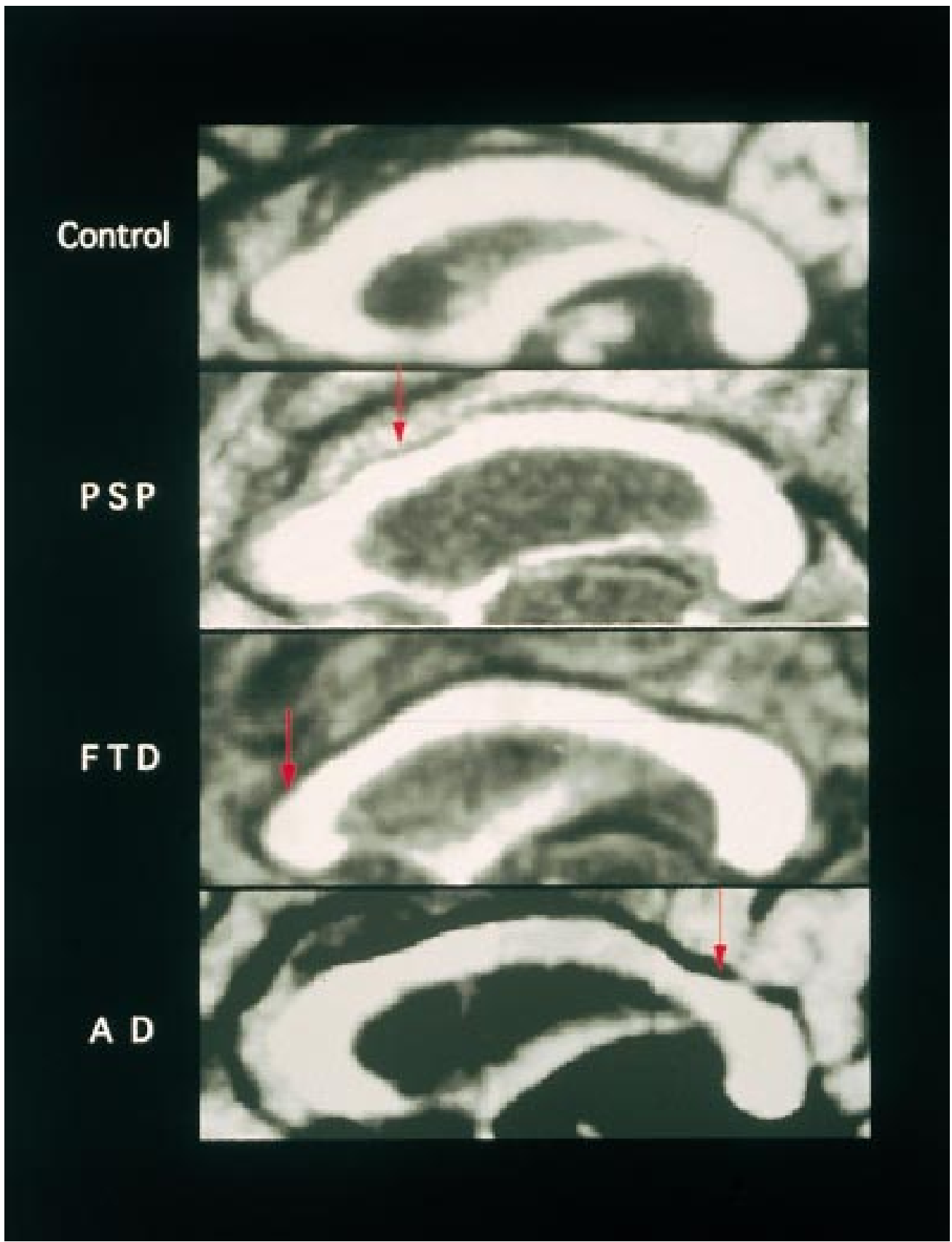

Figure 2 Examples of callosal atrophy. The corpus callosum on T1 weighted MRI images (TR $400 \mathrm{~ms} / \mathrm{TE} 15 \mathrm{~ms}$ ) is shown. Note predominant atrophy of the corpus callosum in the middle-anterior quarter portion in patients with PSP, in the anterior quarter portion in FTD, and in the posterior quarter portion in AD, compared with the control (red arrows).

educational level, and regional size of the corpus callosum as the independent variables. Statistical significance was indicated by $p<0.05$. Statistical analyses were performed using Statview (SAS Institute, Inc, 1998) or SuperANOVA (Abacus Concepts, Inc, 1991) for MacIntosh.

\section{Results}

Patients with PSP were older on average than patients with AD, FTD, and control subjects. However, the age difference among groups was not significant $(p=0.31$, table 1$)$. The MMSE scores were significantly different among the four groups $(F(3,55)=22.6, \mathrm{p}<0.001)$; significantly lower mean MMSE scores were found in patients with PSP, AD, or FTD compared with controls $(p<0.001$ for all, Sheffe's test), but mean MMSE scores in the three patient groups were similar. Duration of disease was significantly different among the three patient groups $(F(2,33)=4.85, \mathrm{p}<0.02)$; significantly longer mean disease duration was seen in patients with PSP compared with patients with $\mathrm{AD}$ and FTD ( $p<0.05$ for both, Sheffe's test), but mean durations in patients with AD and FTD were similar. No significant group differences were seen in sex distribution or level of education.

For the total callosal/skull area ratio, ANOVA showed significant differences among the study groups $(F(3,55)=38.2, \mathrm{p}<0.001)$. The control subjects had larger total callosal/ skull area ratios than the three patient groups 

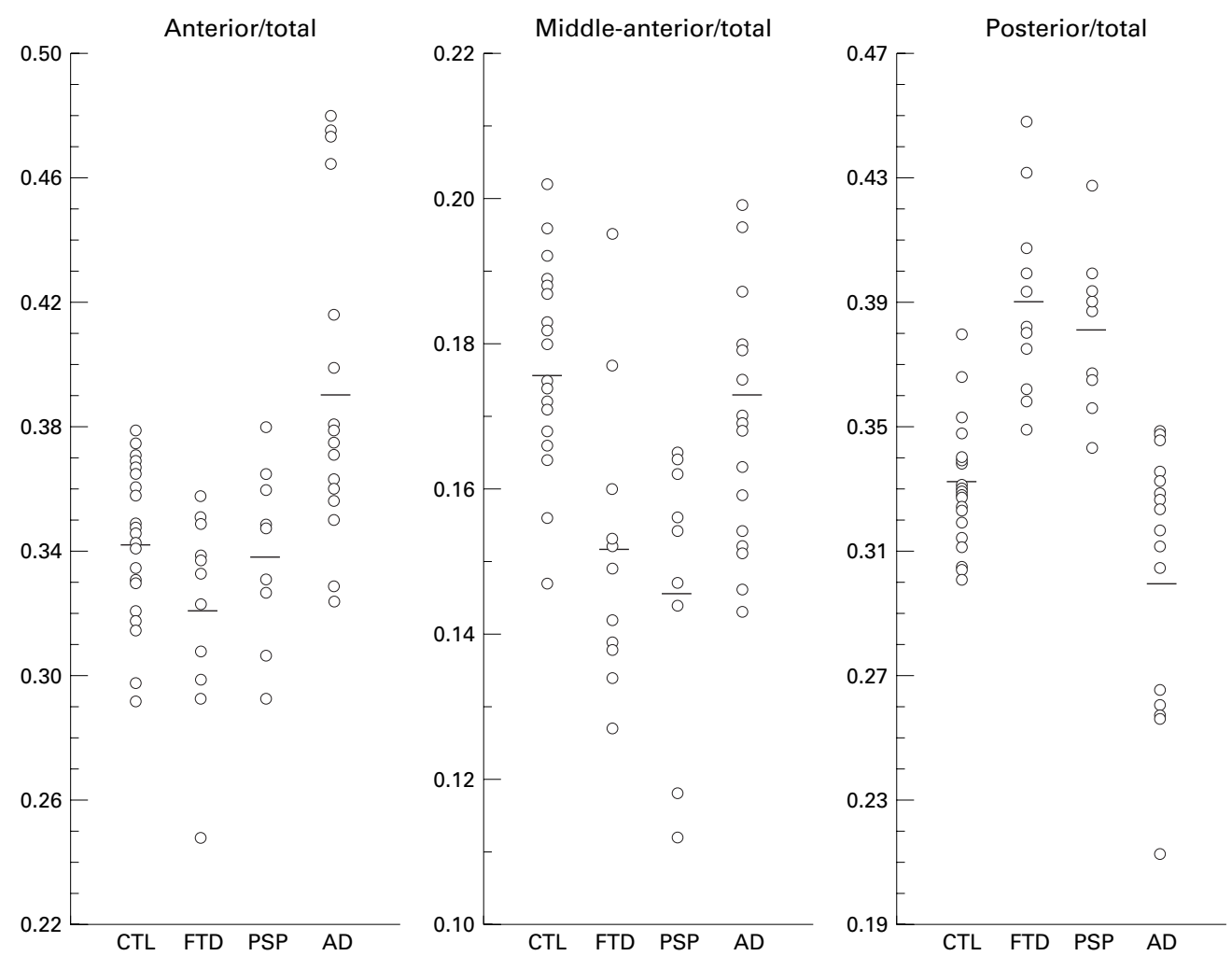

Figure 3 Scatterplots of callosal area/total callosal area ratios of the anterior, middle-anterior, and posterior regions of the corpus callosum. Bars indicate mean values. $C T L=$ controls; $F T D=$ frontotemporal dementia; $P S P=$ progressive supranuclear palsy; $A D=$ Alzheimer's disease.

$(\mathrm{p}<0.001$ for all, table 2). There was a significant interaction between the callosal region and the diagnostic classification $(\mathrm{p}<0.0001$, repeated measures ANOVA), indicating that there was a difference among the study groups across regions (fig 1). When patients with FTD with ALS and those with FTD without ALS were analyzed with repeated measures ANOVA, no significant effect of diagnosis (FTD with ALS $v$ FTD without ALS) on callosal size and no significant interaction between the callosal region and the diagnostic classification were found. In the regional analysis, only the posterior region of patients with PSP showed no significant difference $(p>0.05)$ compared with the controls ( $<<0.001$ for all the other comparisons). Patients with FTD had a significantly smaller callosal/skull area ratio in the anterior region than patients with $\mathrm{AD}(\mathrm{p}<0.001)$ and patients with PSP $(p=0.04)$ and in the middle-anterior region than patients with $\mathrm{AD}(\mathrm{p}=0.03)$. There was no significant difference between the patients with AD and those with PSP.

ANCOVA with the total callosal area/skull area ratio as the covariate showed that the total callosal area/skull area ratio was a significant covariate for each region $(\mathrm{p}<0.001$ for all). The diagnostic classification (FTD, PSP, AD, and

Table 3 Multiple linear regression analysis with the score of the mini mental state examination as the dependent variable

\begin{tabular}{llllc}
\hline Variable & Coefficient & $S E$ & $t$ Value & $p$ Value \\
\hline Total callosal/skull area ratio (\%) & 4.74 & 0.84 & 5.64 & $<0.0001$ \\
Age (y ) & 0.004 & 0.092 & 0.047 & 0.96 \\
Education (y) & 0.11 & 0.24 & 0.43 & 0.67 \\
\hline
\end{tabular}

controls) was a significant factor for each region $(p<0.002)$, except for the middle-posterior region $(\mathrm{p}=0.15)$. Post hoc analysis with contrast methods showed that the adjusted anterior callosal/skull area ratio in FTD ( $\mathrm{p}<0.05 v$ PSP; $\mathrm{p}<0.0005 v \mathrm{AD} ; \mathrm{p}<0.005 v$ controls), the adjusted middle-anterior callosal/skull area ratio in PSP $(\mathrm{p}<0.05 v$ FTD, $\mathrm{p}<0.0005 v \mathrm{AD}$, $\mathrm{p}=0.001 v$ controls), and the adjusted posterior callosal/skull area ratio in $\mathrm{AD}(\mathrm{p}=0.0001 v$ FTD and PSP, $\mathrm{p}<0.05 v$ controls) were significantly smaller than in all of the other three groups (two patient groups and controls), when the total callosal area/skull area ratio was controlled for. To assess more practically whether this characteristic pattern of callosal atrophy (fig 2) is helpful in diagnosis, we calculated regional callosal area/ total callosal area ratios in each group (fig 3). The posterior/total callosal area ratio had little overlap between FTD and AD.

Across all subjects, the total callosal/skull area ratio was significantly correlated with the MMSE scores $(r=0.61, \mathrm{p}<0.001)$. This relation was unchanged after controlling for age and educational level (table 3). Among four callosal regions, only the middle-anterior callosal/skull area ratio showed a significant relation with the MMSE scores, when age, educational level, and callosal/skull area ratios in the other three regions were controlled for (coefficient, 23.9; SE 0.70; p<0.02).

\section{Discussion}

This study showed that the pattern of atrophy of the corpus callosum is different among patients with FTD, PSP, or early onset AD. We 
found that the patients with FTD, PSP, or AD had significantly decreased total callosal/skull area ratios compared with controls, indicating that callosal atrophy is not a specific finding to any of these diseases. However, ANCOVA adjusted for the total callosal area/skull area ratio showed that each disease had a specific pattern of callosal atrophy which may be characteristic of each disease among other degenerative dementias exhibiting callosal atrophy. The predominance of the degree of callosal atrophy in the anterior quarter area in FTD, in the middle-anterior quarter area in PSP, and in the posterior quarter area in $\mathrm{AD}$ differentiated each disease from the others. These patterns of callosal atrophy were consistent with those of cortical hypometabolism in each disease.

Although use of the NINCDS-ADRDA criteria for $\mathrm{AD}$ and the Lund and Manchester criteria for FTD may result in reasonable accuracy for their diagnosis, FTD and AD may be difficult to distinguish clinically. ${ }^{9}$ Therefore, the diagnosis of dementia may require a multidisciplinary approach. Patients with FTD typically have early non-cognitive behavioural changes with relatively spared cognition, frontal atrophy, and enlargement of the sylvian fissures on CT and MRI, and frontotemporal deficits on SPECT or PET. By contrast, patients with $\mathrm{AD}$ have early cognitive changes with relatively preserved personality and behaviour, hippocampal and medial temporal lobe atrophy on CT or MRI, and parietotemporal SPECT or PET deficits. ${ }^{14}$ In this study, FTD and AD were diagnosed based on clinical symptoms and signs. Neither MRI nor SPECT or PET data were used to differentiate three patient groups. Nevertheless, parietotemporal hypometabolism on PET was present in all patients with $\mathrm{AD}$ and bilateral frontal deficits on PET or SPECT were present in all cases of FTD. On the other hand, the difference between the pattern of cortical atrophy in FTD and $\mathrm{AD}$ was not so clear as the difference between the pattern of deficits on PET or SPECT. Differential patterns of cerebral degeneration in FTD and AD have been studied by using MRI or spectroscopy. ${ }^{22-25}$ A study with three dimensional images demonstrated that frontal and anterior temporal atrophy is a distinctive feature of FTD and distinguishes it from $\mathrm{AD}{ }^{25}$ On the routine $\mathrm{MR}$ images, this pattern of cortical atrophy was suspected in all our patients with FTD, but some patients with $\mathrm{AD}$ also showed atrophy in the frontal lobe. In addition, atrophy of the medial temporal region was present in most patients with FTD as well as in all patients with $\mathrm{AD}$. Thus, in the patients studied, the pattern of callosal atrophy was better at differentiating FTD and AD than the pattern of cortical atrophy evaluated by simple visual inspection of scans (data not shown). One study also contrasted callosal atrophy in patients with FTD and patients with $\mathrm{AD}$ who were older than the patients in this study and showed that patients with FTD had a much smaller anterior callosal region than patients with $\mathrm{AD}^{23}$ The evaluation of callosal atrophy may provide additional diagnostic clues to dementias, especially for FTD.
Few studies have compared relative cortical atrophy between patients with FTD and those with PSP by using structural imaging. Frontotemporal dementia and PSP are characterised by different degrees of decline in frontal lobe functions. ${ }^{810}$ Cortical pathologies contribute to frontal lobe dysfunction in FTD, whereas both cortical and subcortical pathologies contribute to PSP. In both FTD and PSP, the callosal atrophy with an anterior predominance seen in this study may reflect cortical pathologies. Thus, more severe atrophy of the anterior quarter region in FTD than in PSP suggests that involvement of the prefrontal cortex is more predominant in FTD than in PSP, because the anterior quarter region includes the commissural fibres originating from the prefrontal cortex. ${ }^{26}$ This interpretation is consistent with previous pathological and clinical reports. Apparent disappearance of neurons in layers 2 and 3 in the prefrontal cortex, including the orbitofrontal cortex, has been demonstrated in FTD, ${ }^{7}$ whereas neurofibrillary degeneration may be prominent in the posterior region in the frontal cortex in PSP. ${ }^{15-17}$ Clinically, behavioural disorders associated with disturbance of prefrontal circuits are more prominent in FTD than in PSP. ${ }^{8}{ }^{10}$ A functional imaging study with PET also showed that FTD showed more severe frontal metabolic impairment than PSP, especially in the frontopolar prefrontal regions. $^{27}$

Some limitations and methodological issues need to be considered. Firstly, we included stroke free patients with headache or dizziness rather than healthy community volunteers as a control group. We did not think this a major problem as they were otherwise neurologically normal. Secondly, it is uncertain whether severity of dementia was matched among the three patient groups, although mean MMSE scores in the three patient groups were similar. The possible difference of severity of dementia might partly account for the difference of callosal size, but its effect on the difference of the pattern of callosal atrophy might be small. Thirdly, the patients with AD and FTD with typical findings on PET or SPECT were analyzed in this study, although the definite diagnosis requires confirmation by neuropathological evaluation. In $\mathrm{AD}$, several MRI studies have shown a decrease in size of the corpus callosum compared with normal controls, but there is inconsistency of the findings about the relative degree of regional atrophy. ${ }^{11} 23$ 27-31 The AD process may be heterogeneous, ${ }^{32}{ }^{33}$ and the severity and distribution of callosal atrophy may vary according to the pathological changes in the cerebral neocortex. Thus, the posterior predominance of the degree of callosal atrophy in $\mathrm{AD}$ seen in this study might be related to the selection of patients with early onset, the minimal white matter changes on MRI, and the pattern of parietotemporal hypometabolism on PET, although a previous study with a larger number of patients also demonstrated the posterior predominance of the degree of callosal atrophy in $\mathrm{AD}^{30}$ In FTD as well, patients with pathological changes accentuated in the tem- 
poral lobe might show a different pattern of callosal atrophy. ${ }^{34}$ Our patients with FTD included five with ALS, which might have affected the results. However, the pattern of callosal atrophy was similar between patients with FTD with ALS and those with FTD without ALS in this study. One pathological study also showed that although the magnitude of the brain atrophy was much greater in FTD alone than in FTD with ALS, the pattern of atrophy was similar. ${ }^{35}$ Furthermore, cortical pathology in PSP may also show considerable variation. ${ }^{15}$ Such variations in each disease may decrease the sensitivity and specificity of the characteristic pattern of callosal atrophy found in this study in a larger patient sample. Lastly, in cases with mild callosal atrophy in the early stage, the variations of morphology and size of the corpus callosum seen in the normal population might mask the characteristic patterns of callosal atrophy in each disease. In that case, a characteristic pattern of callosal atrophy might emerge from follow up MR evaluations. In any case, the pattern of callosal atrophy may be useful as a unique index which may specifically reflect the pattern of neocortical involvement associated with cognitive impairment in degenerative dementias.

In conclusion, the pattern of atrophy of the corpus callosum is different among patients with FTD, PSP, or early onset AD. Corpus callosum atrophy may reflect the pattern of pathological changes in the cerebral neocortex, which is characteristic of each degenerative dementia. This preliminary study suggests that the evaluation of the pattern of callosal atrophy might provide additional diagnostic clues to degenerative dementias, especially for FTD.

We thank the staff of the Department of Radiology and Nuclear Medicine, Faculty of Medicine, Kyoto University for support and technical assistance.

1 Wible CG, Shenton ME, Hokama H, et al. Prefrontal cortex and schizophrenia. A quantitative magnetic resonance imaging study. Arch Gen Psychiatry 1995;52:279-88.

2 Hof PR, Morrison JH. The cellular basis of cortical disconnection in Alzheimer disease and related dementing condi-
tions. In: Terry RD, Katzman R, Bick KL, eds. Alzheimer tions. In: Terry RD, Katzman R, Bick KL, eds.
disease. New York: Raven Press, 1994:197-229.

3 Innocenti GM. General organization of callosal connections in the cerebral cortex. In: Jones EG, Peters A, eds. Cerebral cortex. 5th ed. New York: Plenum Press, 1986:291-353.

4 Mesulam MM. Large-scale neurocognitive networks and distributed processing for attention, language, and memory. Ann Neurol 1990;28:597-613.

5 Pandya DN, Karol EA, Heilbronn D. The topographical distribution of interhemispheric projections in the corpus callosum of rhesus monkey. Brain Res 1971;32:31-43.

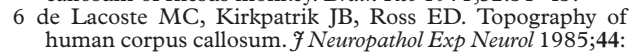
578-91.

7 Clinical and neuropathological criteria for frontotemporal dementia. The Lund and Manchester Groups. F Neurol Neurosurg Psychiatry 1994;57:416-18.

8 Neary D, Snowden JS, Gustafson L, et al. Frontotemporal lobar degeneration: a consensus on clinical diagnostic lobar degeneration: a consensus

9 Lopez OL, Litvan I, Catt KE, et al. Accuracy of four clinical Lopez OL, Litvan I, Catt KE, et al. Accuracy of four clinical
diagnostic criteria for the diagnosis of neurodegenerative diagnostic criteria for the diagnosis of
dementias. Neurology 1996;53:1292-9.

10 Litvan I. Progressive supranuclear palsy revisited. Acta Neurol Scand 1998;98:73-84.
11 Yamauchi H, Fukuyama H, Harada K, et al. Callosal atrophy parallels decreased cortical oxygen metabolism and neuropsychological impairment in Alzheimer's disease. Arch Neurol 1993;50:1070-4.

12 Yamauchi H, Fukuyama H, Ouchi Y, et al. Corpus callosum atrophy in amyotrophic lateral sclerosis. F Neurol Sci 1995; 134:189-96.

13 Yamauchi H, Fukuyama H, Nagahama Y, et al. Atrophy of the corpus callosum, cognitive impairment, and cortical hypometabolism in progressive supranuclear palsy. Ann Nypometabolism in pro $1997 ; 41: 606-14$.

14 Duara R, Barker W, Luis CA. Frontotemporal dementia and Alzheimer's disease: differential diagnosis. Dement Geriatr Cogn Disord 1999;10(suppl 1):37-42.

15 Daniel SE, de Bruin VMS, Lees AJ. The clinical and pathological spectrum of Steele-Richardson-Olszewski syndrome (progressive supranuclear palsy): a reappraisal. Brain 1995;118:759-70.

16 Hof PR, Delacourte A, Bouras C. Distribution of cortical neurofibrillary tangles in progressive supranuclear palsy: a neurofibrillary tangles in progressive supranuclear palsy: a
quantitative analysis of six cases. Acta Neuropathol Berl quantitative an

17 Vermersch P, Robitaille Y, Bernier L, et al. Biochemical mapping of neurofibrillary degeneration in a case of progressive supranuclear palsy: evidence for general cortical involvement. Acta Neuropathol Berl 1994;87:572-7.

18 Folstein MF, Folstein SE. A mini-mental state: a practical method for grading the cognitive state of patients for the clinician. F. Psychiatr Res 1975;12:189-93.

19 Litvan I, Agid Y, Jankovic J, et al. Accuracy of clinical criteria for the diagnosis of progressive supranuclear palsy ria for the diagnosis of progressive supranuclear palsy
(Steele-Richardson-Olszewski syndrome). Neurology 1996; 46:922-30.

20 McKhann G, Drachman D, Flostein M, et al. Clinical diagnosis of Alzheimer's disease: report of the NINCDSADRDA work group under the auspices of department of Health and Human services task force on Alzheimer's disease. Neurology 1984;34:939-44.

21 Laissy JP, Patrux B, Duchateau C, et al. Midsagittal MR measurement of the corpus callosum in healthy subjects and diseased patients: a prospective survey. AfNR Am $\mathcal{F}$ Neuroradiol 1993;14:145-54.

22 Frisoni GB, Beltramello A, Geroldi C, et al. Brain atrophy in frontotemporal dementia. F Neurol Neurosurg Psychiatry 1996;61:157-65.

23 Kaufer DI, Miller BL, Itti L, et al. Midline cerebral morphometry distinguishes frontotemporal dementia and Alzheimer's disease. Neurology 1997;48:978-85.

24 Ernst T, Chang L, Melchor R, et al. Frontotemporal dementia and early Alzheimer disease: differentiation with frontal lobe H-1 MR spectroscopy. Radiology 1997;203:829-36.

25 Kitagaki H, Mori E, Yamaji S, et al. Frontotemporal dementia and Alzheimer's disease: evaluation of cortical atrophy with automated hemispheric surface display generated with MR images. Radiology 1998;208:431-9.

26 Barbas H, Pandya DN. Topography of commissural fibers of the prefrontal cortex in the rhesus monkey. Exp Brain Res 1984;55:187-91

27 Garraux G SE, Degueldre C, Lemaire C, et al. Comparison of impaired subcortico-frontal metabolic networks in normal aging, subcortico-frontal dementia, and cortical frontal dementia. Neuroimage 1999;10:149-62.

28 Biegon A, Eberling JL, Richardson BC, et al. Human corpus callosum in aging and Alzheimer's disease: a magnetic resonance imaging study. Neurobiol Aging 1994;15:393-7.

29 Janowsky JS, Kaye JA, Carper RA. Atrophy of the corpus callosum in Alzheimer's disease versus healthy aging. $7 \mathrm{Am}$ Geriatr Soc 1996;44:798-803

30 Lyoo IK, Satlin A, Lee CK, et al. Regional atrophy of the corpus callosum in subjects with Alzheimer's disease and multi-infarct dementia. Psychiatry Res 1997;74:63-72.

31 Hampel H, Teipel SJ, Alexander GE, et al. Corpus callosum atrophy is a possible indicator of region and cell type-specific neuronal degeneration in Alzheimer disease: a magnetic resonance imaging analysis. Arch Neurol 1998;55: mag-8.

32 Holzer M, Holzapfel HP, Zedlick D, et al. Abnormally phosphorylated tau protein in Alzheimer's disease: heterogeneity of individual regional distribution and relationship to clinical severity. Neuroscience 1994;63:499-516.

33 Waldemar G, Bruhn P, M. K, Johnsen A, et al. Heterogeneity of neocortical cerebral blood flow deficits in dementia of the Alzheimer type: a [ $\left.{ }^{99 \mathrm{~m}} \mathrm{Tc}\right]-\mathrm{d}, \mathrm{l}-\mathrm{HMPAO}$ SPECT study. F Neurol Neurosurg Psychiatry 1994;57:285-95.

34 Edwards-Lee T, Miller BL, Benson DF, et al. The temporal variant of frontotemporal dementia. Brain 1997;120:102740.

35 Mann DM, South PW. The topographic distribution of brain atrophy in frontal lobe dementia. Acta Neuropathol 1993;85:334-40. 